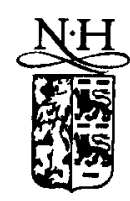

ELSEVIER
11 September 1997

PHYSICS LETTERS B

\title{
Cross section of hadron production in $\gamma \gamma$ collisions at LEP
}

\section{L3 Collaboration}

M. Acciarri ${ }^{\mathrm{ac}}$, O. Adriani ${ }^{\mathrm{r}}$, M. Aguilar-Benitez ${ }^{\mathrm{ab}}, \mathrm{S}$. Ahlen ${ }^{\ell}$, J. Alcaraz ${ }^{\mathrm{ab}}$, G. Alemanni $^{\mathrm{x}}$, J. Allaby ${ }^{\mathrm{s}}$, A. Aloisio ${ }^{\mathrm{ae}}$, G. Alverson ${ }^{\mathrm{m}}$, M.G. Alviggi ${ }^{\mathrm{ae}}$, G. Ambrosi ${ }^{\mathrm{u}}, \mathrm{H}$. Anderhub ${ }^{\mathrm{az}}$, V.P. Andreev ${ }^{\text {g,an }}$, T. Angelescu ${ }^{\text {n}}$, F. Anselmo ${ }^{\mathrm{j}}$, A. Arefiev ${ }^{\text {ad }}$, T. Azemoon ${ }^{\mathrm{c}}$, T. Aziz $^{\mathrm{k}}$, P. Bagnaia ${ }^{\text {am }}$, L. Baksay ${ }^{\text {at }}$, S. Banerjee ${ }^{k}$, Sw. Banerjee ${ }^{k}$, K. Banicz ${ }^{\text {av }}$, A. Barczyk ${ }^{\text {azaw }}$, R. Barillère ${ }^{\mathrm{s}}$, L. Barone ${ }^{\text {am }}$, P. Bartalini ${ }^{\text {aj }}$, A. Baschirotto ${ }^{\text {ac }}$, M. Basile ${ }^{\mathrm{j}}$, R. Battiston ${ }^{\text {aj, }}$, A. Bay ${ }^{x}$, F. Becattini ${ }^{r}$, U. Becker ${ }^{q}$, F. Behner ${ }^{a z}$, J. Berdugo ${ }^{\text {ab }}$, P. Berges ${ }^{q}$, B. Bertucci ${ }^{\text {aj }}$, B.L. Betev ${ }^{\text {az }}$, S. Bhattacharya ${ }^{k}$, M. Biasini ${ }^{\text {s }}$, A. Biland ${ }^{\text {az }}$, G.M. Bilei ${ }^{\text {aj, J.J. Blaising }}{ }^{d}$, S.C. Blyth ${ }^{\text {ak }}$, G.J. Bobbink ${ }^{\text {b }}$, R. Bock ${ }^{a}$, A. Böhm ${ }^{\text {a }}$, L. Boldizsar ${ }^{\circ}$, B. Borgia ${ }^{\text {am }}$, D. Bourilkov ${ }^{\text {az }}$, M. Bourquin ${ }^{\text {u, S. Braccini }}{ }^{\text {u }}$, J.G. Branson ${ }^{\text {ap }}$, V. Brigljevic ${ }^{\text {az }}$, I.C. Brock ${ }^{\text {ak }}$, A. Buffini ${ }^{r}$, A. Buijs ${ }^{\text {au }}$, J.D. Burger ${ }^{q}$, W.J. Burger ${ }^{\text {u }}$, J. Busenitz ${ }^{\text {at }}$, A. Button ${ }^{c}$, X.D. Cai ${ }^{q}$, M. Campanelli ${ }^{\text {az }}$, M. Capell ${ }^{\mathrm{q}}$, G. Cara Romeo ${ }^{\mathrm{j}}$, G. Carlino ${ }^{\mathrm{ae}}$, A.M. Cartacci ${ }^{\mathrm{r}}$, J. Casaus ${ }^{\mathrm{ab}}$, G. Castellini ${ }^{\mathrm{r}}$, F. Cavallari ${ }^{\text {am }}$, N. Cavallo ${ }^{\text {ae }}$, C. Cecchi ${ }^{\text {}}$, M. Cerrada ${ }^{\text {ab }}$, F. Cesaroni ${ }^{y}$, M. Chamizo ${ }^{\text {ab }}$, Y.H. Chang ${ }^{\text {bb }}$, U.K. Chaturvedi t, S.V. Chekanov ${ }^{\text {ag }}$, M. Chemarin ${ }^{\text {aa }}$, A. Chen ${ }^{\text {bb }}$, G. Chen ${ }^{\text {h }}$, G.M. Chen ${ }^{\text {h }}$, H.F. Chen ${ }^{v}$, H.S. Chen ${ }^{\text {h }}$, X. Chereau ${ }^{\text {d }}$, G. Chiefari ${ }^{\text {ae }}$, C.Y. Chien ${ }^{\text {e }}$, L. Cifarelli ${ }^{\text {ao }}$, F. Cindolo ${ }^{j}$, C. Civinini ${ }^{\text {r }}$, I. Clare ${ }^{q}$, R. Clare ${ }^{q}$, H.O. Cohn ${ }^{\text {ah }}$, G. Coignet ${ }^{\text {d }}$, A.P. Colijn ${ }^{\text {b }}$, N. Colino ${ }^{\text {ab }}$, V. Commichau ${ }^{a}$, S. Costantini ${ }^{i}$, F. Cotorobai ${ }^{n}$, B. de la Cruz ${ }^{\text {ab }}$, A. Csilling ${ }^{\circ}$, T.S. Dai ${ }^{q}$, R. D'Alessandro ${ }^{r}$, R. de Asmundis ${ }^{\text {ae }}$, A. Degré ${ }^{d}$, K. Deiters ${ }^{\text {aw }}$, D. della Volpe ${ }^{\text {ae }}$, P. Denes ${ }^{a \ell}$, F. DeNotaristefani ${ }^{\text {am }}$, D. DiBitonto ${ }^{\text {at }}$, M. Diemoz ${ }^{\text {am }}$, D. van Dierendonck ${ }^{\text {b }}$, F. Di Lodovico ${ }^{\text {az }}$, C. Dionisi ${ }^{\text {am }}$, M. Dittmar ${ }^{\text {az }}$, A. Dominguez ${ }^{\text {ap }}$, A. Doria ${ }^{\text {ae }}$, M.T. Dova ${ }^{\mathrm{t}, 4}$, D. Duchesneau ${ }^{\mathrm{d}}$, P. Duinker ${ }^{\mathrm{b}}$, I. Duran ${ }^{\mathrm{aq}}$, S. Dutta ${ }^{k}$, S. Easo ${ }^{\text {aj }}$, Yu. Efremenko ${ }^{\text {ah }}$, H. El Mamouni aa , A. Engler ${ }^{\text {ak }}$, F.J. Eppling ${ }^{\mathrm{q}}$, F.C. Erné ${ }^{\text {, }}$ J.P. Ernenwein ${ }^{\text {aa }}$, P. Extermann ${ }^{u}$, M. Fabre ${ }^{\text {aw }}$, R. Faccini ${ }^{\text {am }}$, S. Falciano ${ }^{\text {am }}$, A. Favara ${ }^{\text {r }}$ J. Fay ${ }^{\text {aa }}$, O. Fedin ${ }^{\text {an }}$, M. Felcini ${ }^{\text {az }}$, B. Fenyi ${ }^{\text {at }}$, T. Ferguson ${ }^{\text {ak }}$, F. Ferroni ${ }^{a m}$, H. Fesefeldt ${ }^{\text {a }}$, E. Fiandrini ${ }^{\text {aj }}$, J.H. Field ${ }^{\text {u }}$, F. Filthaut ${ }^{\text {ak }}$, P.H. Fisher ${ }^{\text {q }}$, I. Fisk ${ }^{\text {ap }}$, G. Forconi ${ }^{q}$, L. Fredj ${ }^{u}$, K. Freudenreich ${ }^{\text {az }}$, C. Furetta ${ }^{\text {ac }}$, Yu. Galaktionov ${ }^{\text {ad, }}$, S.N. Ganguli ${ }^{k}$, P. Garcia-Abia ${ }^{a y}$, S.S. Gau ${ }^{\mathrm{m}}$, S. Gentile ${ }^{\mathrm{am}}$, N. Gheordanescu ${ }^{\mathrm{n}}$, S. Giagu ${ }^{\text {am }}$, S. Goldfarb ${ }^{\mathrm{x}}$, J. Goldstein ${ }^{\ell}$, Z.F. Gong ${ }^{\vee}$, A. Gougas ${ }^{\mathrm{e}}$, G. Gratta ${ }^{\text {ai }}$, M.W. Gruenewald ${ }^{\mathrm{i}}$, V.K. Gupta ${ }^{\mathrm{a} \ell}$, A. Gurtu ${ }^{\text {}}$, L.J. Gutay ${ }^{\text {av }}$, B. Hartmann ${ }^{a}$, A. Hasan ${ }^{\text {af }}$, D. Hatzifotiadou ${ }^{j}$, T. Hebbeker ${ }^{i}$,

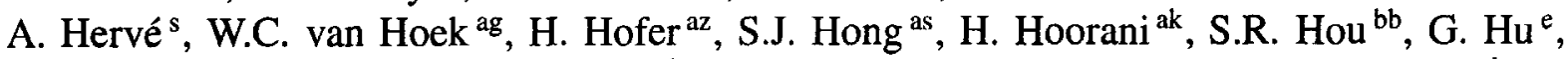
V. Innocente ${ }^{\mathrm{s}}$, K. Jenkes ${ }^{\mathrm{a}}$, B.N. Jin ${ }^{\mathrm{h}}$, L.W. Jones ${ }^{\mathrm{c}}$, P. de Jong ${ }^{\mathrm{s}}$, I. Josa-Mutuberria ${ }^{\text {ab }}$, 
A. Kasser ${ }^{\text {x }}$, R.A. Khan ${ }^{\text {, D. Kamrad }}{ }^{\text {ay }}$, Yu. Kamyshkov ${ }^{\text {ah }}$, J.S. Kapustinsky ${ }^{\text {, }}$, Y. Karyotakis ${ }^{\text {d }}$, M. Kaur ${ }^{\text {t.5 }}$, M.N. Kienzle-Focacci ${ }^{\text {}}$, D. Kim ${ }^{\text {am }}$, D.H. Kim ${ }^{\text {as }}$, J.K. Kim ${ }^{\text {as }}$, S.C. Kim ${ }^{\text {as }}$, Y.G. Kim ${ }^{\text {as }}$, W.W. Kinnison ${ }^{z}$, A. Kirkby ai , D. Kirkby ${ }^{\text {ai }}$, J. Kirkby ${ }^{\text {s }}$, D. Kiss ${ }^{\circ}$, W. Kittel ${ }^{\text {ag }}$, A. Klimentov q,ad , A.C. König ${ }^{\text {ag }}$, A. Kopp ${ }^{\text {ay }}$, I. Korolko ${ }^{\text {ad }}$, V. Koutsenko ${ }^{\text {q,ad }}$, R.W. Kraemer ${ }^{\text {ak }}$,W. Krenz ${ }^{\text {a }}$, A. Kunin ${ }^{\text {q,ad }}$, P. Ladron de Guevara ${ }^{\text {ab }}$, I. Laktineh ${ }^{\text {aa }}$, G. Landi ${ }^{\mathrm{r}}$, C. Lapoint ${ }^{\mathrm{q}}$, K. Lassila-Perini ${ }^{\text {az }}$, P. Laurikainen ${ }^{\mathrm{w}}$, M. Lebeau ${ }^{\mathrm{s}}$, A. Lebedev ${ }^{\mathrm{q}}$, P. Lebrun ${ }^{\text {aa }}$, P. Lecomte ${ }^{\text {az }}$, P. Lecoq ${ }^{\text {s }}$, P. Le Coultre ${ }^{\text {az }}$, J.M. Le Goff ${ }^{\text {, }}$, R. Leiste ${ }^{\text {ay }}$, E. Leonardi am , P. Levtchenko ${ }^{\text {an }}$, C. Li $^{\text {v }}$, C.H. Lin ${ }^{\text {bb }}$, W.T. Lin ${ }^{\text {bb }}$, F.L. Linde ${ }^{\text {b,s }}$, L. Lista ${ }^{\text {ae }}$, Z.A. Liu ${ }^{\text {h}}$, W. Lohmann ${ }^{\text {ay }}$, E. Longo ${ }^{\text {am }}$, W. Lu ${ }^{\text {ai }}$, Y.S. Lu ${ }^{\text {h }}$, K. Lübelsmeyer ${ }^{a}$, C. Luci ${ }^{\text {am }}$, D. Luckey ${ }^{q}$, L. Luminari ${ }^{\text {am }}$, W. Lustermann ${ }^{\text {aw }}$, W.G. Ma ${ }^{\mathrm{v}}$, M. Maity ${ }^{\mathrm{k}}$, G. Majumder ${ }^{\mathrm{k}}$, L. Malgeri $^{\text {am }}$, A. Malinin ${ }^{\text {ad }}$, C. Maña ${ }^{\text {ab }}$, D. Mangeol ${ }^{\mathrm{ag}}$, S. Mangla ${ }^{\mathrm{k}}$, P. Marchesini ${ }^{\mathrm{az}}$, A. Marin ${ }^{\ell}$, J.P. Martin aa , F. Marzano ${ }^{\text {am }}$, G.G.G. Massaro ${ }^{\text {b }}$, D. McNally ${ }^{\text {s }}$, R.R. McNeil ${ }^{\text {, }}$, S. Mele ${ }^{\text {ae }}$, L. Merola ae ${ }^{\text {ae }}$ M. Meschini ${ }^{\text {r }}$, W.J. Metzger ${ }^{\text {ag }}$, M. von der Mey ${ }^{\mathrm{a}}$, Y. Mi ${ }^{\mathrm{x}}$, A. Mihul ${ }^{\text {n }}$, A.J.W. van Mil ${ }^{\text {ag }}$, G. Mirabelli am ${ }^{\text {, J. Mnich }}{ }^{\text {s }}$, P. Molnar ${ }^{\mathrm{i}}$, B. Monteleoni ${ }^{\mathrm{r}}$, R. Moore ${ }^{c}$, S. Morganti ${ }^{a m}$, T. Moulik ${ }^{k}$, R. Mount ${ }^{\text {ai }}$, S. Müller ${ }^{a}$, F. Muheim ${ }^{\text {u, }}$ A.J.M. Muijs ${ }^{b}$, S. Nahn ${ }^{\text {q }}$, M. Napolitano ${ }^{\text {ae }}$, F. Nessi-Tedaldi az ${ }^{\text {az }}$ H. Newman ${ }^{\text {ai }}$, T. Niessen ${ }^{\text {a }}$, A. Nippe ${ }^{\text {a }}$, A. Nisati am , H. Nowak ${ }^{\text {ay }}$, Y.D. Oh as , H. Opitz ${ }^{\text {a }}$, G. Organtini am, R. Ostonen ${ }^{\text {w }}$, C. Palomares $^{\text {ab }}$, D. Pandoulas ${ }^{\text {a }}$, S. Paoletti ${ }^{\text {am }}$, P. Paolucci ${ }^{\text {ae }}$, H.K. Park ${ }^{\text {ak }}$, I.H. Park ${ }^{\text {as }}$, G. Pascale ${ }^{a m}$, G. Passaleva ${ }^{\mathrm{r}}$, S. Patricelli ${ }^{\text {ac }}$, T. Paul ${ }^{\mathrm{m}}$, M. Pauluzzi ${ }^{\text {aj }}$, C. Paus ${ }^{\mathrm{a}}$, F. Pauss ${ }^{\text {az }}$, D. Peach ${ }^{\text {s }}$, Y.J. Pei ${ }^{a}$, S. Pensotti ${ }^{\text {ac }}$, D. Perret-Gallix ${ }^{d}$, B. Petersen ${ }^{\text {ag }}$, S. Petrak ${ }^{i}$, A. Pevsner ${ }^{\mathrm{e}}$, D. Piccolo ${ }^{\mathrm{ae}}$, M. Pieri ${ }^{\mathrm{r}}$, J.C. Pinto ${ }^{\text {ak }}$, P.A. Piroué ${ }^{\mathrm{a} \ell}$, E. Pistolesi ${ }^{\text {ac }}$, V. Plyaskin ${ }^{\text {ad }}$, M. Pohl ${ }^{\text {az }}$, V. Pojidaev ad,r, H. Postema ${ }^{\text {a }}$, N. Produit ${ }^{\text {u, D. Prokofiev a }}$, G. Rahal-Callot ${ }^{\text {az }}$, N. Raja ${ }^{\text {k }}$, P.G. Rancoita ${ }^{\text {ac }}$, M. Rattaggi ${ }^{\text {ac }}$, G. Raven ${ }^{\text {ap }}$, P. Razis ${ }^{a f}$, K. Read ${ }^{\text {ah }}$, D. Ren ${ }^{\text {az }}$, M. Rescigno ${ }^{\text {am }}$, S. Reucroft ${ }^{\mathrm{m}}$, T. van Rhee ${ }^{\text {au }}$, S. Riemann ${ }^{\text {ay }}$, K. Riles ${ }^{c}$, A. Robohm ${ }^{\text {az }}$, J. Rodin ${ }^{q}$, B.P. Roe ${ }^{c}$, L. Romero ${ }^{\text {ab }}$, S. Rosier-Lees ${ }^{d}$, Ph. Rosselet ${ }^{\mathrm{x}}$, W. van Rossum ${ }^{\text {au }}$, S. Roth ${ }^{\mathrm{a}}$, J.A. Rubio ${ }^{\mathrm{s}}$, D. Ruschmeier ${ }^{\mathrm{i}}$, H. Rykaczewski ${ }^{\text {az }}$, J. Salicio ${ }^{\text {s }}$, E. Sanchez ${ }^{\text {ab }}$, M.P. Sanders ${ }^{\text {ag }}$, M.E. Sarakinos ${ }^{\text {w }}$, S. Sarkar ${ }^{\text {}}$, M. Sassowsky ${ }^{a}$, C. Schäfer ${ }^{\text {a }}$, V. Schegelsky ${ }^{\text {an }}$, S. Schmidt-Kaerst ${ }^{a}$, D. Schmitz ${ }^{a}$, P. Schmitz ${ }^{a}$, N. Scholz ${ }^{\text {az }}$, H. Schopper ${ }^{\text {ba }}$, D.J. Schotanus ${ }^{\text {ag }}$, J. Schwenke ${ }^{\text {a }}$, G. Schwering ${ }^{\text {a }}$, C. Sciacca ${ }^{\text {ae }}$, D. Sciarrino ${ }^{u}$, L. Servoli aj, S. Shevchenko ${ }^{\text {ai }}$, N. Shivarov ${ }^{\text {ar }}$, V. Shoutko ${ }^{a d}$, J. Shukla ${ }^{\text {z }}$, E. Shumilov ${ }^{\text {ad }}$, A. Shvorob ${ }^{\text {ai }}$, T. Siedenburg a ${ }^{\text {a }}$ D. Son ${ }^{\text {as }}$, A. Sopczak ${ }^{\text {ay }}$, B. Smith ${ }^{q}$, P. Spillantini ${ }^{r}$, M. Steuer ${ }^{q}$, D.P. Stickland ${ }^{a \ell}$, A. Stone ${ }^{g}$, H. Stone ${ }^{a \ell}$, B. Stoyanov ${ }^{a r}$, A. Straessner ${ }^{a}$, K. Strauch ${ }^{p}$, K. Sudhakar ${ }^{k}$, G. Sultanov ${ }^{t}$, L.Z. Sun ${ }^{\text {v }}$, G.F. Susinno ${ }^{\text {u, H. Suter }}{ }^{\text {az }}$, J.D. Swain ${ }^{\mathrm{t}}$, X.W. Tang ${ }^{\mathrm{h}}$, L. Tauscher ${ }^{\mathrm{f}}$, L. Taylor ${ }^{\mathrm{m}}$, Samuel C.C. Ting ${ }^{q}$, S.M. Ting ${ }^{q}$, M. Tonutti ${ }^{a}$, S.C. Tonwar ${ }^{\mathrm{k}}$, J. Tóth ${ }^{\circ}$, C. Tully ${ }^{a}{ }^{\text {, }}$, H. Tuchscherer ${ }^{\text {at }}$, K.L. Tung ${ }^{\mathrm{h}}$, Y. Uchida ${ }^{\mathrm{q}}$, J. Ulbricht ${ }^{\mathrm{az}}$, U. Uwer ${ }^{\mathrm{s}}$, E. Valente ${ }^{\mathrm{am}}$, R.T. Van de Walle ${ }^{\text {ag }}$, G. Vesztergombi ${ }^{\circ}$, I. Vetlitsky ${ }^{\text {ad }}$, G. Viertel ${ }^{\text {az }}$, M. Vivargent ${ }^{d}$, R. Völkert ${ }^{\text {ay }}, H$. Vogel ${ }^{\text {ak }}, H$. Vogt ${ }^{\text {ay }}$, I. Vorobiev ${ }^{\text {ad }}$, A.A. Vorobyov an, A. Vorvolakos ${ }^{\text {af }}$, M. Wadhwa ${ }^{\mathrm{f}}$, W. Wallraff ${ }^{\mathrm{a}}$, J.C. Wang ${ }^{\mathrm{q}}$, X.L. Wang ${ }^{\mathrm{v}}$, Z.M. Wang ${ }^{\mathrm{v}}$, A. Weber ${ }^{\mathrm{a}}$,

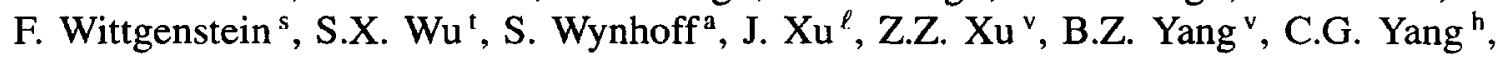




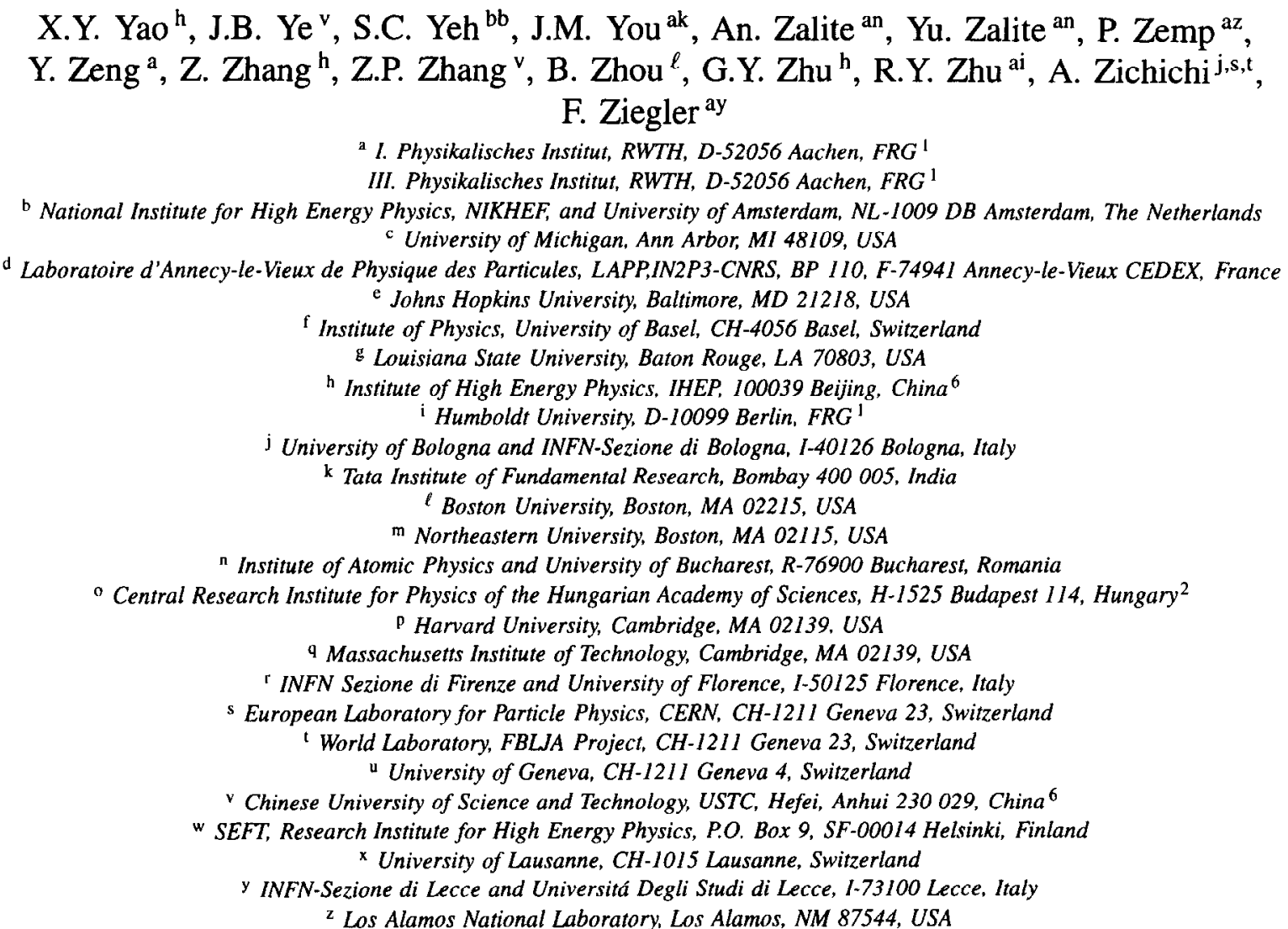

aa Institut de Physique Nucléaire de Lyon, IN2P3-CNRS, Université Claude Bernard, F-69622 Villeurbanne, France ab Centro de Investigaciones Energeticas, Medioambientales y Tecnologicas, CIEMAT, E-28040 Madrid, Spain ${ }^{3}$ ac INFN-Sezione di Milano, I-20133 Milan, Italy

ad Institute of Theoretical and Experimental Physics, ITEP, Moscow, Russia

ae INFN-Sezione di Napoli and University of Naples, 1-80125 Naples, Italy

${ }^{\text {af }}$ Department of Natural Sciences, University of Cyprus, Nicosia, Cyprus

ag University of Nijmegen and NIKHEF, NL-6.525 ED Nijmegen. The Netherlands

ah Oak Ridge National Laboratory, Oak Ridge, TN 37831, USA

ai California Institute of Technology, Pasadena, CA 91125, USA

aj INFN-Sezione di Perugia and Universitá Degli Studi di Perugia, 1-06100 Perugia, Italy

ak Carnegie Mellon University, Pittsburgh, PA 15213, USA

al Princeton University, Princeton, NJ 08544, USA

am INFN-Sezione di Roma and University of Rome, "La Sapienza", I-00185 Rome, Italy

an Nuclear Physics Institute, St. Petersburg, Russia

ao University and INFN, Salerno, I-84100 Salerno, Italy

ap University of California, San Diego, CA 92093, USA

aq Dept. de Fisica de Particulas Elementales, Univ. de Santiago, E-15706 Santiago de Compostela, Spain

ar Bulgarian Academy of Sciences, Central Lab. of Mechatronics and Instrumentation, BU-1113 Sofia, Bulgaria

as Center for High Energy Physics, Korea Adv. Inst. of Sciences and Technology, 305-701 Taejon, South Korea

at University of Alabama, Tuscaloosa, AL 35486, USA

au Utrecht University and NIKHEF, NL-3584 CB Utrecht, The Netherlands

av Purdue University, West Lafayette, IN 47907, USA

aw Paul Scherrer Institut, PSI, CH-5232 Villigen, Switzerland

ay DESY-Institut für Hochenergiephysik, D-15738 Zeuthen, FRG

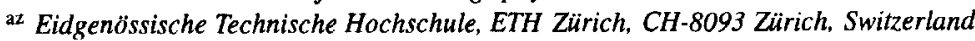


ba University of Hamburg, D-22761 Hamburg, FRG

bb High Energy Physics Group, Taiwan, ROC

Received 5 May 1997

Editor: K. Winter

\begin{abstract}
The reaction $\mathrm{e}^{+} \mathrm{e}^{-} \rightarrow \mathrm{e}^{+} \mathrm{e}^{-} \gamma^{*} \gamma^{*} \rightarrow \mathrm{e}^{+} \mathrm{e}^{-}$hadrons is analysed using data collected by the $\mathrm{L} 3$ detector during the LEP runs at $\sqrt{s}=130-140 \mathrm{GeV}$ and $\sqrt{s}=161 \mathrm{GeV}$. The cross sections $\sigma\left(\mathrm{e}^{+} \mathrm{e}^{-} \rightarrow \mathrm{e}^{+} \mathrm{e}^{-}\right.$hadrons $)$and $\sigma(\gamma \gamma \rightarrow$ hadrons $)$ are measured in the interval $5 \leq W_{\gamma \gamma} \leq 75 \mathrm{GeV}$. The energy dependence of the $\sigma(\gamma \gamma \rightarrow$ hadrons) cross section is consistent with the universal Regge behaviour of total hadronic cross sections. (c) 1997 Published by Elsevier Science B.V.
\end{abstract}

\section{Introduction}

At high energies the two-photon process $\mathrm{e}^{+} \mathrm{e}^{-} \rightarrow$ $\mathrm{e}^{+} \mathrm{e}^{-} \gamma^{*} \gamma^{*} \rightarrow \mathrm{e}^{+} \mathrm{e}^{-}$hadrons is a copious source of hadron production. In this reaction most of the initial energy is taken by the scattered electrons and positrons. As their scattering angle is close to the beam they often go undetected. The variable $Q^{2}$ is defined by the four-momentum transfer squared from the beam to one of the scattered electrons: $Q^{2}=-q^{2}$. If one of the scattered electrons is measured in forward detectors the event is said to be tagged. The hadron system has predominantly a low mass value. A large part of the hadrons escape detection, due to the large diffractive cross section and to the Lorentz boost of the $\gamma \gamma$ system. For these events, the measured effective mass $W_{\text {vis }}$ is smaller than the centre of mass energy of the two interacting photons $W_{\gamma \gamma}$. For high values of $\sqrt{s}$ the $W_{\text {vis }}$ spectrum of twophoton processes is well separated from that of the $\mathrm{e}^{+} \mathrm{e}^{-}$annihilation processes.

A photon can interact as a point-like particle (direct component Fig. 1a). Often a quantum fluctuation transforms the photon into a vector meson $\rho, \omega, \phi, \ldots$

\footnotetext{
' Supported by the German Bundesministerium für Bildung, Wissenschaft, Forschung und Technologie.

2 Supported by the Hungarian OTKA fund under contract numbers T14459 and T24011.

${ }^{3}$ Supported also by the Comisión Interministerial de Ciencia y Technología.

${ }^{4}$ Also supported by CONICET and Universidad Nacional de La Plata, CC 67, 1900 La Plata, Argentina.

5 Also supported by Panjab University, Chandigarh-160014, India.

${ }^{6}$ Supported by the National Natural Science Foundation of China.
}

a)

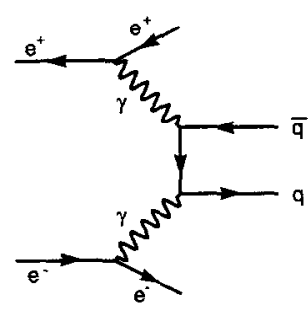

c)

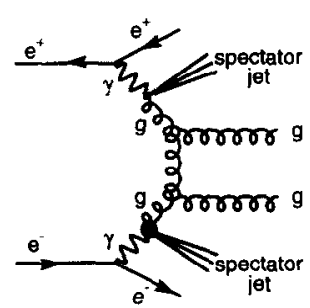

b)

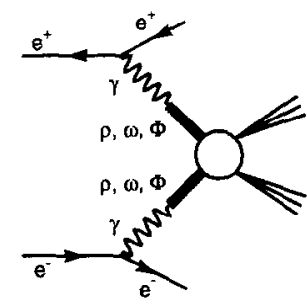

d)

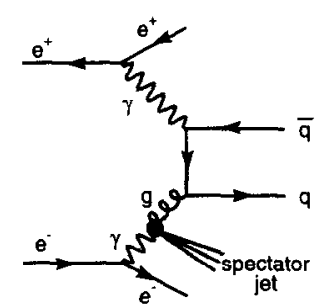

Fig. 1. Some diagrams contributing to $\gamma \gamma \rightarrow$ hadrons reactions: a) direct b) VMD c) double resolved d) single resolved.

(VMD component Fig. 1b), opening up all the possibilities of hadronic interactions (Regge poles, Pomeron exchange, etc.). In hard scattering the structure of the photon can be resolved into quarks and gluons. Some examples are given in Fig. 1c and 1d. The relative amounts of these components and their respective properties are not yet fully understood. Recently there has been an effort by Schuler and Sjöstrand [1] and by Engel and Ranft [2] (Dual Parton Model) to construct a model consistent with the knowledge accumulated from $\gamma \mathrm{p}$, ep and pp scattering 
data.

Both groups have provided a Monte Carlo generator which can be compared with the data. In PYTHIA [3] where both incoming photons are assumed to be on the mass shell, we have complemented the code by generating the photon flux in the Equivalent Photon Approximation [4] with a cutoff $Q^{2} \leq m_{\rho}^{2}$. The model is then valid only for events with $Q^{2} \simeq 0$. The Monte Carlo generator PHOJET [2] uses the $\gamma \gamma$ luminosity function, $\mathcal{L}_{\gamma \gamma}$, for transverse photons, taking into account the hadronic couplings of the photon by using a generalised vector dominance model.

In this paper we analyse only data where the scattered electrons are not detected (anti-tagged events). Thus the interacting photons are quasi-real: $\left\langle Q^{2}\right\rangle \simeq$ $0.025 \mathrm{GeV}^{2}$. The visible cross sections and event shape of the data are compared to the Monte Carlo predictions.

The total cross section $\sigma\left(\mathrm{e}^{+} \mathrm{e}^{-} \rightarrow \mathrm{e}^{+} \mathrm{e}^{-}\right.$hadrons $)$is measured for the average $\mathrm{e}^{+} \mathrm{e}^{-}$centre of mass energy of $\sqrt{s}=133 \mathrm{GeV}$ and for $\sqrt{s}=161 \mathrm{GeV}$. The twophoton cross section $\sigma(\gamma \gamma \rightarrow$ hadrons $)$ is then derived in the interval $5 \leq W_{\gamma \gamma} \leq 75 \mathrm{GeV}$. This measurement is compared to previous results obtained for $W_{\gamma \gamma} \leq 10 \mathrm{GeV}$ and fitted with the universal Regge [7] parametrisation of Donnachie and Landshoff [8].

\section{Event selection and comparison with Monte Carlo}

Data have been collected with the L3 detector at $\sqrt{s}=130,136,140 \mathrm{GeV}$ with a total integrated luminosity of $4.98 \mathrm{pb}^{-1}$ during 1995 and at $\sqrt{s}=161 \mathrm{GeV}$ with an integrated luminosity of $10.37 \mathrm{pb}^{-1}$ during 1996.

A detailed description of each subsystem of the L3 detector and its performance is given in [9] and [10] The analysis described in this paper is mainly based on the central tracking system, the high resolution electro-magnetic calorimeter and the hadron calorimeter. Particles scattered at small angles are measured by the luminosity monitors on each side of the detector, covering a polar angle range between 25 and $69 \mathrm{mrad}$.

The events used in this analysis are collectcd prcdominantly by a track trigger [11] which requires at least two charged particles with $p_{t}>150 \mathrm{MeV}$, back to back, in the plane transverse to the beam, within $\pm 41^{\circ}$.

Hadronic two-photon events are selected by the following criteria:

- At least three tracks are required to eliminate the dominant $\mathrm{e}^{+} \mathrm{e}^{-} \rightarrow \mathrm{e}^{+} \mathrm{e}^{-}$leptons channels. A track is defined by a transverse momentum $p_{t}>100 \mathrm{MeV}$, at least 12 wire hits and a distance of closest approach to the nominal vertex smaller than $10 \mathrm{~mm}$ in the transverse plane. With the additional condition that the total energy deposited in the electromagnetic calorimeter exceeds $500 \mathrm{MeV}$, the beamgas and beam-wall backgrounds are suppressed.

- The energy in the electro-magnetic calorimeter is required to be smaller than $30 \mathrm{GeV}$ and the energy deposited in the hadron calorimeter smaller than 20 $\mathrm{GeV}$, to exclude annihilation events.

- An anti-tag condition is imposed which excludes events with energy greater than $30 \mathrm{GeV}$ in the luminosity monitor, in a fiducial polar angle region of $27-64 \mathrm{mrad}$ at $133 \mathrm{GeV}$ and $33-64 \mathrm{mrad}$ at 161 $\mathrm{GeV}$. The fiducial region is smaller at $161 \mathrm{GeV}$ because the inner part of the luminosity detector is shadowed by the shielding inserted into the beam pipe to absorb synchrotron radiation.

The cuts are illustrated in Fig. 2. After selection the background from beam-gas and beam-wall interactions is found to be negligible.

The visible effective mass of the event is calculated from the four-momentum vectors of the measured particles. All particles are assumed to be pions, except for electro-magnetic clusters identified as photons. A cluster in the electro-magnetic calorimeter, with no nearby track in a $200 \mathrm{mrad}$ cone, is recognised as a photon if its energy in the hadron calorimeter is smaller than $20 \%$ of the electro-magnetic energy. Clusters in the hadron calorimeter, without any track in a 300 mrad cone and with an energy greater than $20 \%$ of the electro-magnetic energy are considered as pions, since they are mainly outside the track chamber detection region. Clusters in the luminosity monitor are also included in the calculation of the visible effective mass

$W_{\text {vis }}^{2}=\left(\sum_{i} E_{i}\right)^{2}-\left(\sum_{i} p_{i}\right)^{2} \quad i=$ pions, photons

The analysis is limited to events with $W_{\text {vis }} \geq 5 \mathrm{GeV}$. The number of events selected is 8220 at $\sqrt{s}=133$ $\mathrm{GeV}$ and 22857 at $\sqrt{s}=161 \mathrm{GeV}$. 


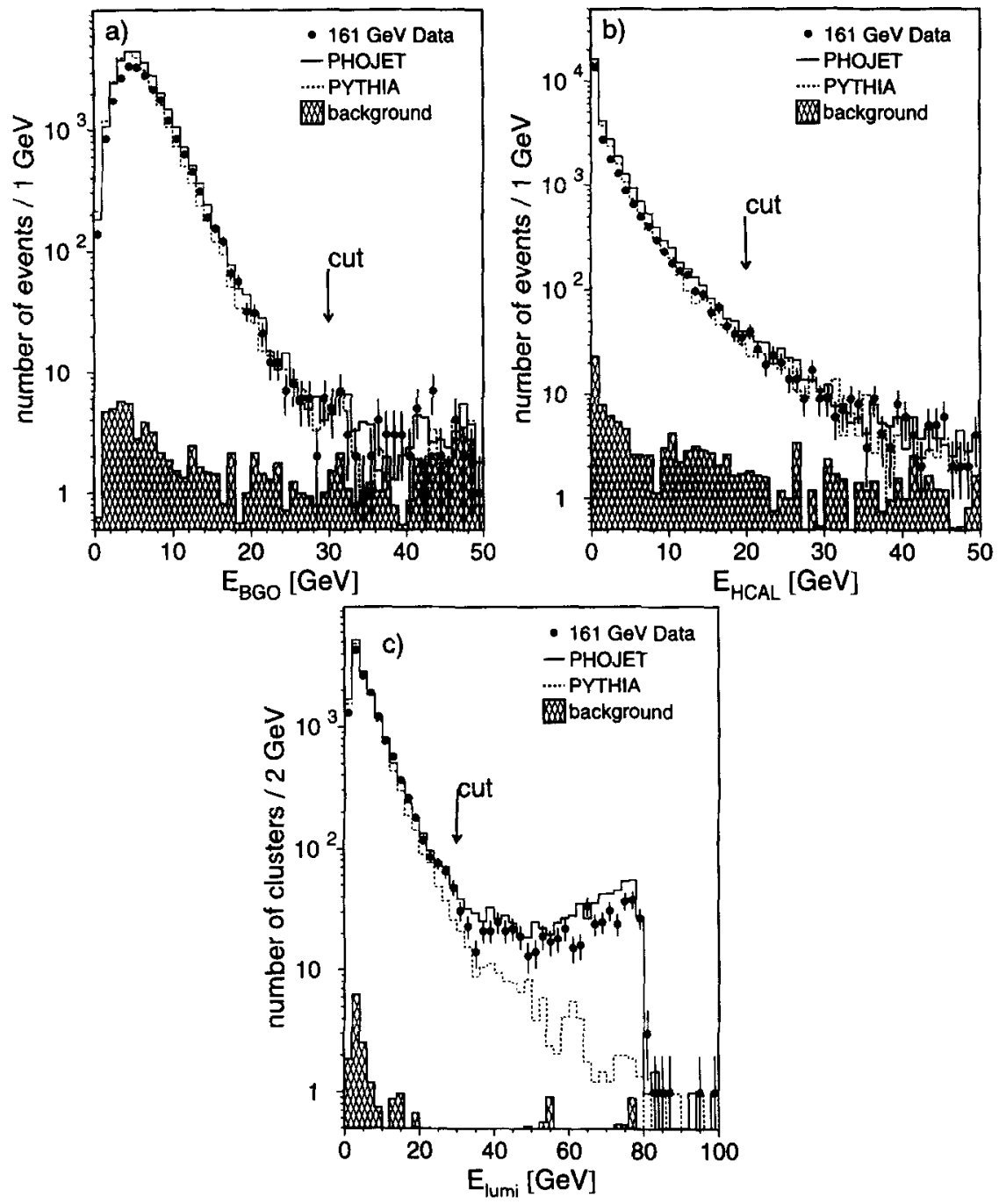

Fig. 2. The energy measured in the calorimeters compared to PHOJET and PYTHIA expectations, a) electro-magnetic BGO calorimeter b) hadronic calorimeter c) luminosity monitor calorimeter. The backgrounds are indicated as a shaded area.

The background, due mainly to annihilation processes and two-photon $\tau$ production, is subtracted from the data. It varies from less than a per cent at a mass of $5 \mathrm{GeV}$ to a few per cent at high masses as can be seen in Fig. 3 where the $W_{\text {vis }}$ spectrum is shown for both energies.

High statistics samples of PHOJET $^{7}$ [2] and PYTHIA $^{8}$ [3] events have been generated for each beam energy. For the annihilation processes $\mathrm{e}^{+} \mathrm{e}^{-} \rightarrow$

\footnotetext{
${ }^{7}$ PHOJET version $1.05 \mathrm{c}$

${ }^{8}$ PYTHIA version 5.718 and JETSET version 7.408
}

hadrons $(\gamma), \operatorname{ZZ}(\gamma)$, Zee $(\gamma)$, We $\nu(\gamma)$ we have simulated events with PYTHIA [3], and we have used KORALZ [5] for $\mathrm{e}^{+} \mathrm{e}^{-} \rightarrow \tau^{+} \tau^{-}(\gamma)$. For the $\mathrm{e}^{+} \mathrm{e}^{-} \rightarrow$ $\mathrm{e}^{+} \mathrm{e}^{-} \tau^{+} \tau^{-}$channel we have simulated events with DIAG36 [6].

The events were simulated in the $\mathrm{L} 3$ detector using GEANT [12] and GEISHA [13] programs and passed through the same reconstruction program as the data. The trigger inefficiency was taken into account during the simulation. It was studied with two-photon and Bhabha events by comparing the response of the 

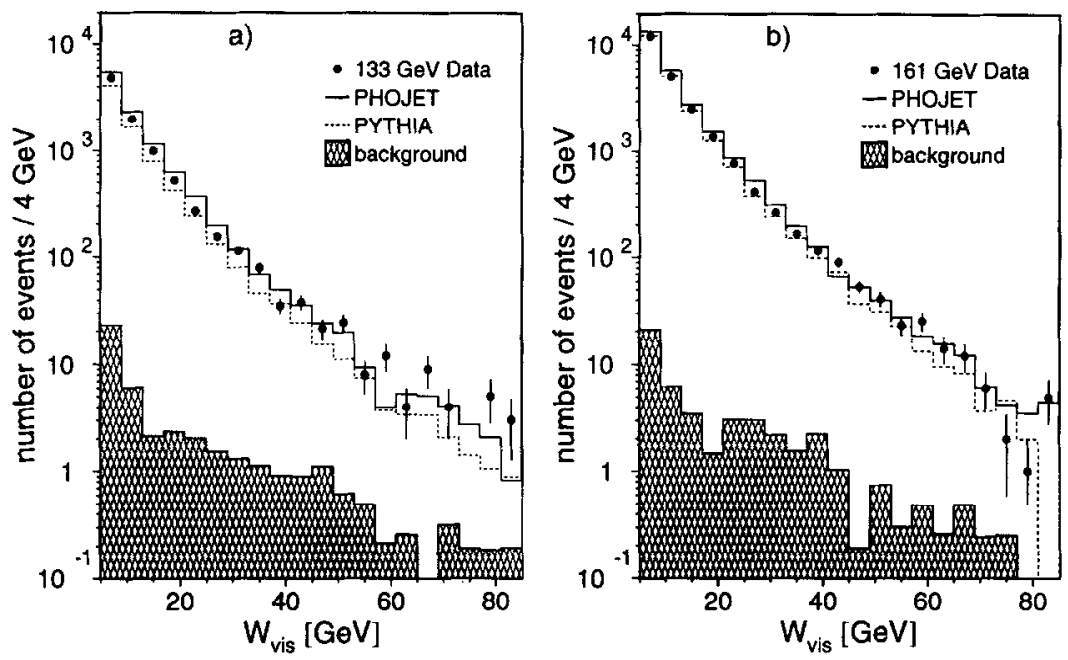

Fig. 3. The measured visible mass a) at $\sqrt{s}=133 \mathrm{GeV}$ b) at $\sqrt{s}=161 \mathrm{GeV}$, compared to PHOJET and PYTHIA expectations. The backgrounds are indicated as a shaded area.

Table 1

Number of selected hadronic events with $W_{\mathrm{vis}} \geq 5 \mathrm{GeV}$ as a function of the minimum number of tracks required. The Monte Carlo events are normalized to the luminosity of the data.

\begin{tabular}{llccccc}
\hline & & Data & PYTHIA & Data/PYTHIA & PHOJET & Data/PHOJET \\
\hline \multirow{2}{*}{$130-140 \mathrm{GeV}$} & $\geq 3$ tracks & 8220 & 8682 & 0.94 & 9400 & 0.87 \\
& $\geq 4$ tracks & 6786 & 7643 & 0.89 & 8346 & 0.81 \\
$161 \mathrm{GeV}$ & $\geq 5$ tracks & 5307 & 6045 & 0.88 & 6788 & 0.78 \\
& $\geq 3$ tracks & 22857 & 23161 & 0.99 & 25826 & 0.89 \\
& $\geq 4$ tracks & 19573 & 20454 & 0.96 & 23082 & 0.85 \\
& $\geq 5$ tracks & 15525 & 16338 & 0.95 & 18888 & 0.82 \\
\hline
\end{tabular}

track trigger to the response of the calorimetric energy triggers. It was found that $(93 \pm 1) \%$ of the events with $W_{\text {vis }} \geq 5 \mathrm{GeV}$ are accepted by the trigger. The number of expected events are given in Table 1. The absolute normalisation of PHOJET gives about $10 \%$ higher values than PYTHIA. The Monte Carlo predictions for electro-magnetic and hadron calorimeter total encrgy agree well with the data as shown in Fig. 2. A variation of the cuts inside $\pm 10 \mathrm{GeV}$ shows that the ratio of accepted events in the data and in the Monte Carlo remains stable within $1 \%$. The energy distribution in the luminosity monitor (Fig. 2c) shows a good agreement for the low energy values, i.e. for the hadronic component inside the detector. When the scattered electron or positron reaches the detector, the agreement is maintained with the PHOJET Monte Carlo, while these configurations are missing in PYTHIA because of the cutoff $Q^{2} \leq m_{\rho}^{2}$ in the event generation.

The visible mass spectra are rather well reproduced by the generators at both centre of mass energies (Fig. 3). In Fig. 4 the longitudinal and transverse momentum of the hadronic system, normalised to its energy, are shown. The longitudinal momentum distribution is not in good agreement with both Monte Carlo simulations whereas the mean value of the energy as a function of the polar angle (Fig. 5) for tracks, photons in the electro-magnetic calorimeter and isolated clusters in the hadron calorimeter agrees with the Monte Carlo expectations. A detailed study of the longitudinal momentum distribution shows that the region at the edges $\left(\left|p_{\text {long }} / E_{\mathrm{vis}}\right|>0.6\right)$ is mainly correlated to low values of $W_{\text {vis }}$ while the high values 

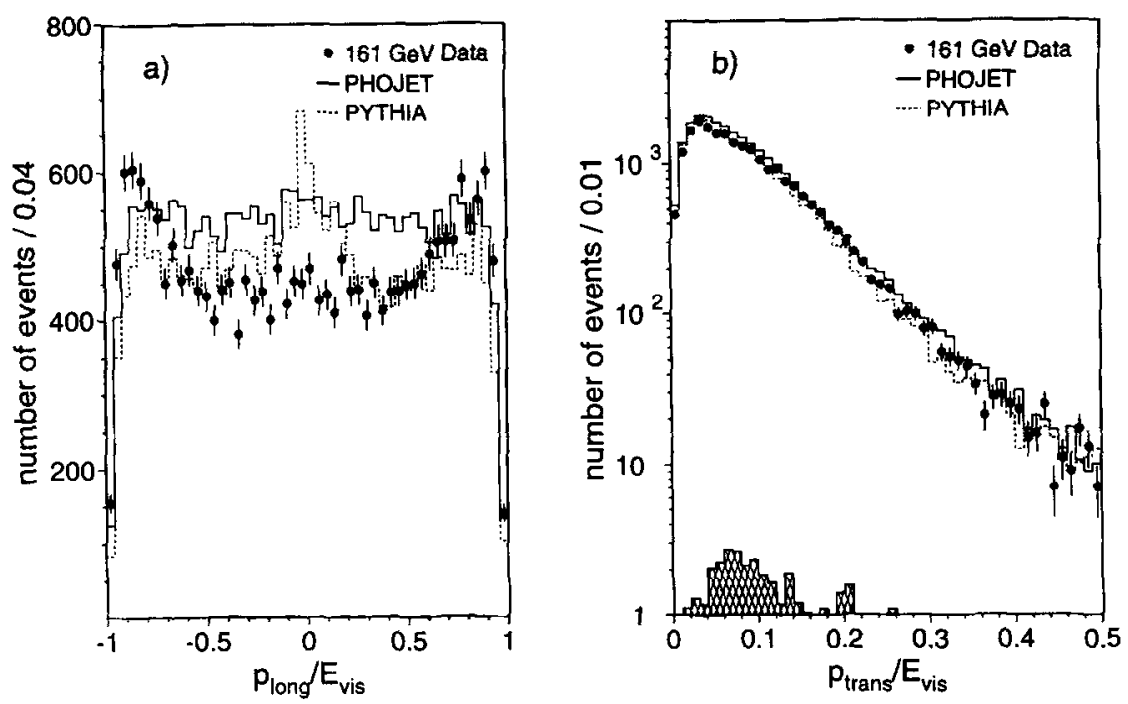

Fig. 4. Longitudinal (a) and transverse (b) momentum of the hadronic system normalized to the visible energy. The data are compared to PHOJET and PYTHIA expectations.

of $W_{\text {vis }}$ are in the central $p_{\text {long }} / E_{\text {vis }}$ region.

The transverse momentum distribution of the tracks is compared in Fig. 6 for four different mass intervals; the agreement is satisfactory. The charged track multiplicity however is not well modelled as can be seen in Table 1 . Since the cut on the number of tracks affects the measurement of the cross sections, the full analysis is repeated for a lower cut of 3,4 and 5 tracks. The variation of the cross sections, thus obtained, is included in the systematic errors.

In conclusion some features of the distributions are not well reproduced by the two generators. The disagreement between data and Monte Carlo does not exceed $30 \%$ and it is of the same order as the disagreement between the two generators. The differences between the two Monte Carlo simulations are used to estimatc the systcmatic errors.

\section{Measurement of cross sections}

\subsection{Unfolding and efficiency}

From the observed distribution of the visible effective mass, $W_{\text {vis }}$, the true hadron mass $W_{\gamma \gamma}$ distribution must be extracted. The number of observed events are then corrected for the efficiency and acceptance of the detector. The two steps are illustrated in Fig. 7a by using PHOJET Monte Carlo events.

The measured $W_{\text {vis }}$ spectrum is weakly correlated to the total centre of mass energy of the $\gamma \gamma$ system because a large part of the produced particles go undetected in the forward and backward regions. In order to obtain the $W_{y \gamma}$ distribution, subdivided in ten $i$ intervals, from the $W_{\text {vis }}$ spectrum, subdivided in twenty $j$-intervals, the following unfolding relation is used:

$W_{\gamma \gamma}(i)=\sum_{j=1}^{n} A_{i j} W_{\mathrm{vis}}(j)$

The matrix $A_{i j}$ is constructed by considering for each Monte Carlo event the measured $W_{\text {vis }}$ and its generated $W_{\gamma \gamma}$ value as follows:

$A_{i j}=\frac{P\left(W_{\mathrm{vis}}(j) \mid W_{\gamma \gamma}(i)\right) P\left(W_{\gamma \gamma}(i)\right)}{\sum_{l} P\left(W_{\mathrm{vis}}(j) \mid W_{\gamma \gamma}(l)\right) P\left(W_{\gamma \gamma}(l)\right)}$

where $P\left(W_{\text {vis }} \mid W_{\gamma \gamma}\right)$ is the likelihood of observing the measured $W_{\text {vis }}$ given a generated $W_{\gamma \gamma}$ value and $P\left(W_{\gamma \gamma}\right)$ is the initially generated $W_{\gamma \gamma}$ distribution after acceptance and efficiency cuts (dashed line in Fig. 7a).

After unfolding, the events are corrected for detector acceptance and efficiency using the ratio between selected and generated events in each $W_{\gamma \gamma}$ interval (Fig. 7b). This includes geometrical effects as well as inefficiencies of the detector, of the trigger and of the 

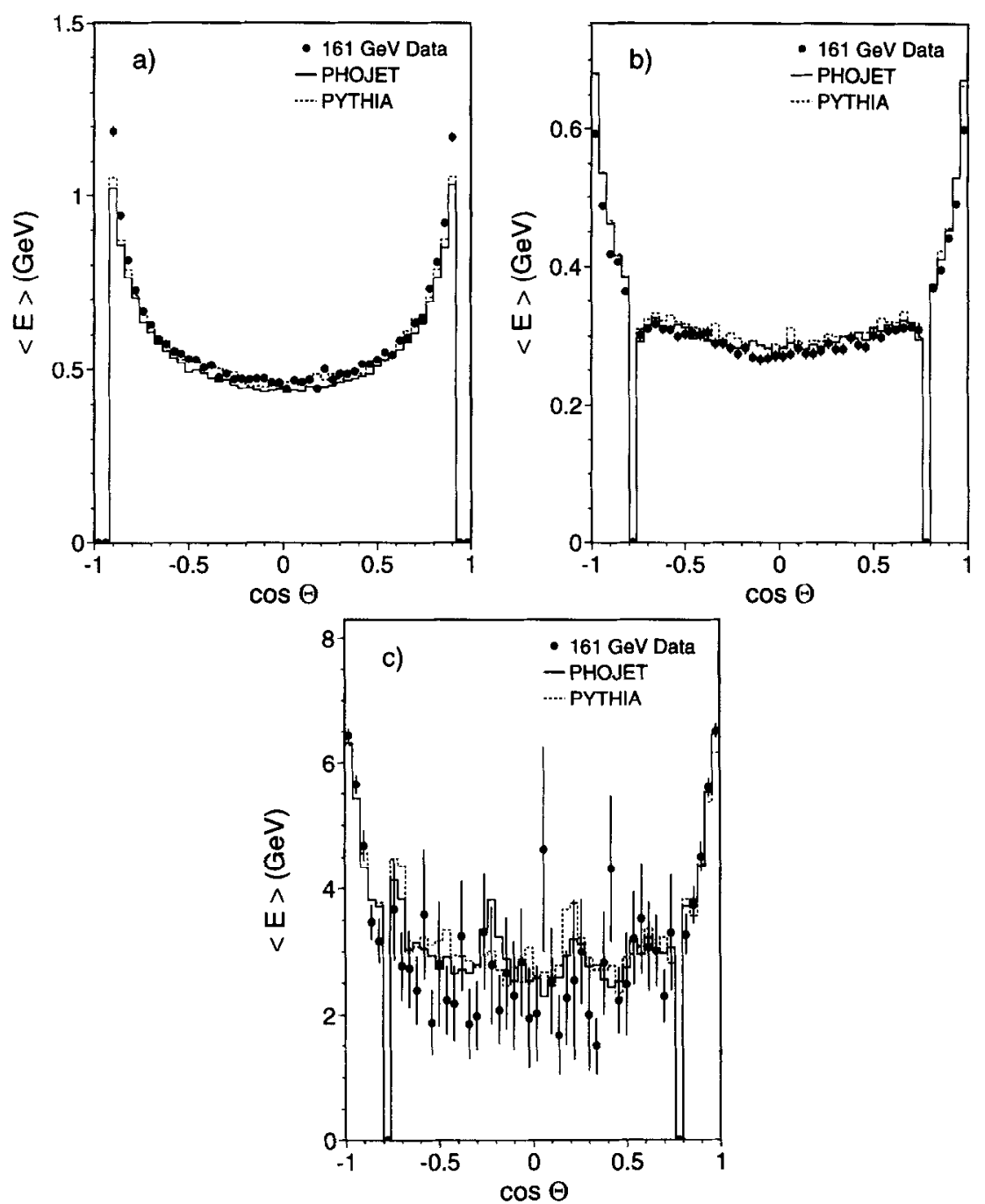

Fig. 5. (a) The mean energy of tracks, (b) of electro-magnetic clusters, (c) of hadron calorimeter clusters as a function of the polar angle. The data are compared to PHOJET and PYTHIA expectations.

analysis. The low acceptance below $W_{\gamma \gamma}=20 \mathrm{GeV}$ is due to the $W_{\text {vis }}$ cutoff of $5 \mathrm{GeV}$. For $W_{\gamma \gamma}>20 \mathrm{GeV}$, the acceptance is rather constant.

This method relies on a good modelling of the data and demands a high statistics Monte Carlo sample. Unfolding methods have been widely discussed in Ref. [14]. Two methods recently developed by D'Agostini [15] and by Höcker and Kartvelishvili [16] produce similar results.

\subsection{Cross sections and systematic errors}

From the number of events, corrected with the PHOJET Monte Carlo in each $W_{\gamma \gamma}$ bin, and the integrated $\mathrm{e}^{+} \mathrm{e}^{-}$luminosity, the cross section $d \sigma\left(\mathrm{e}^{+} \mathrm{e}^{-} \rightarrow\right.$ $\mathrm{e}^{+} \mathrm{e}^{-}$hadrons) is measured. The results are listed in Table 2 and the differcntial cross section $d \sigma / d W_{\gamma \gamma}$ is shown in Fig. 8a. The fast decrease of the cross section as a function of $W_{\gamma \gamma}$ is due to the two photon luminosity function, $\mathcal{L}_{\gamma \gamma}$, which depends on $W_{\gamma \gamma}^{2} / s$.

Unfolding introduces a strong correlation in the measurement, the correlation matrix is given in Table 

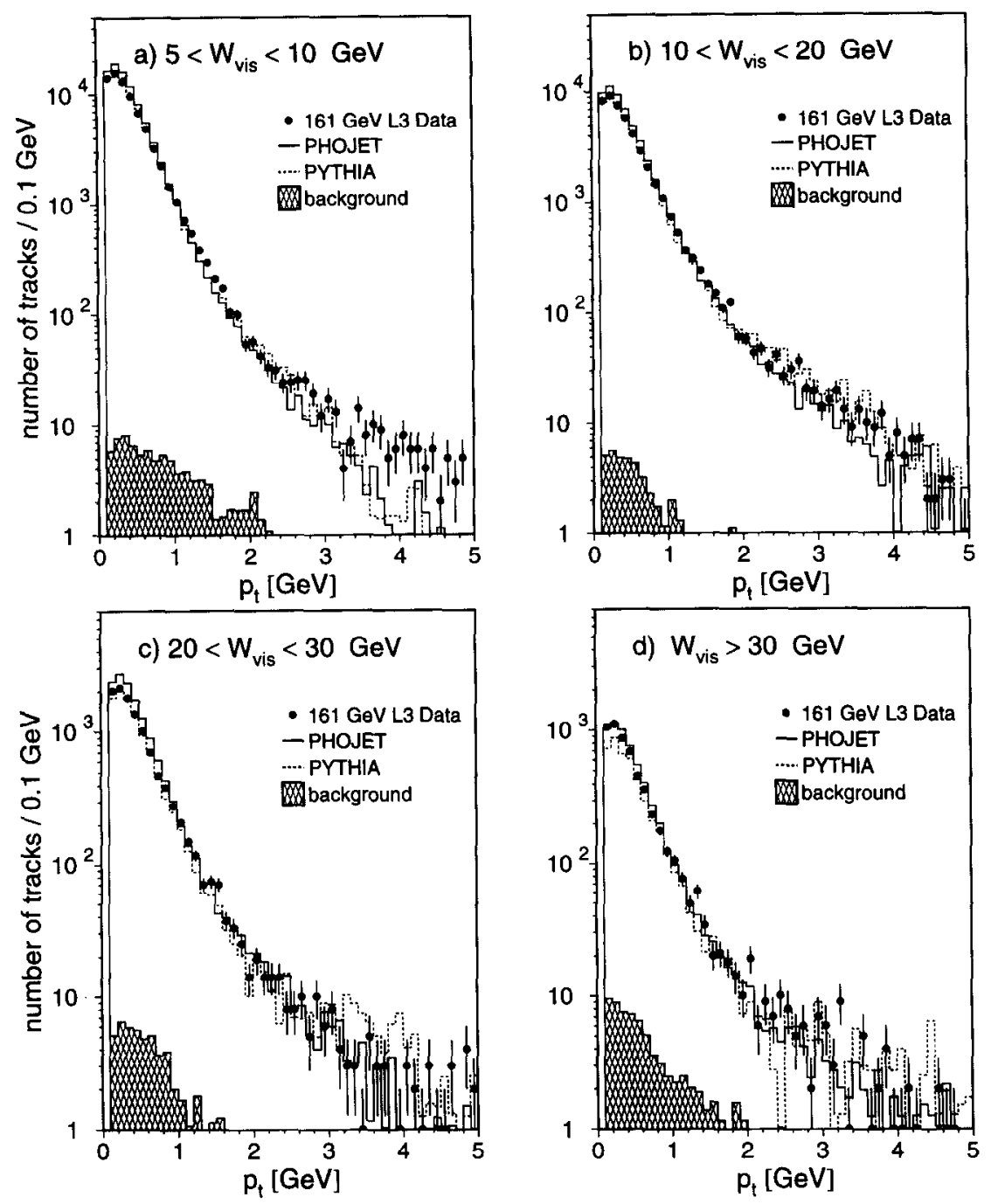

Fig. 6. The transverse momentum $p_{t}$ distribution of tracks compared to PHOJET and PYTHIA expectations in four visible mass intervals. The background is indicated as a shaded area.

3 . The square-root of the diagonal elements of the error matrix are given in Table 2 as statistical errors. The uncertainties due to the data statistics dominate over the uncertainties of the unfolding matrix due to Monte Carlo statistics.

In order to evaluate the systematic errors related to the model, the full analysis is repeated with PYTHIA. Both analyses are also repeated for a minimum number of four and five tracks. In evaluating the systematic errors the effects which produce a mass dependent error are separated from those giving only a normalisation shift. The main sources of systematic errors are:
1. differences between data and Monte Carlo in the representation of the hadronic showers in the hadron calorimeter and in the small angle luminosity monitor. For the energy deposited in the hadron calorimeter no significant discrepancy (Fig. 5c) is observed, while there is a $6 \%$ difference in the average value of the energy deposited at small angles (Fig. 2c). Such a shift can produce a mass dependent variation $\Delta \sigma / \sigma \simeq$ $\pm 0.002 W_{\text {vis }}(\mathrm{GeV})$ in the cross section.

2. the use of PYTHIA instead of PHOJET in the analysis gives a bin-to-bin difference which is 

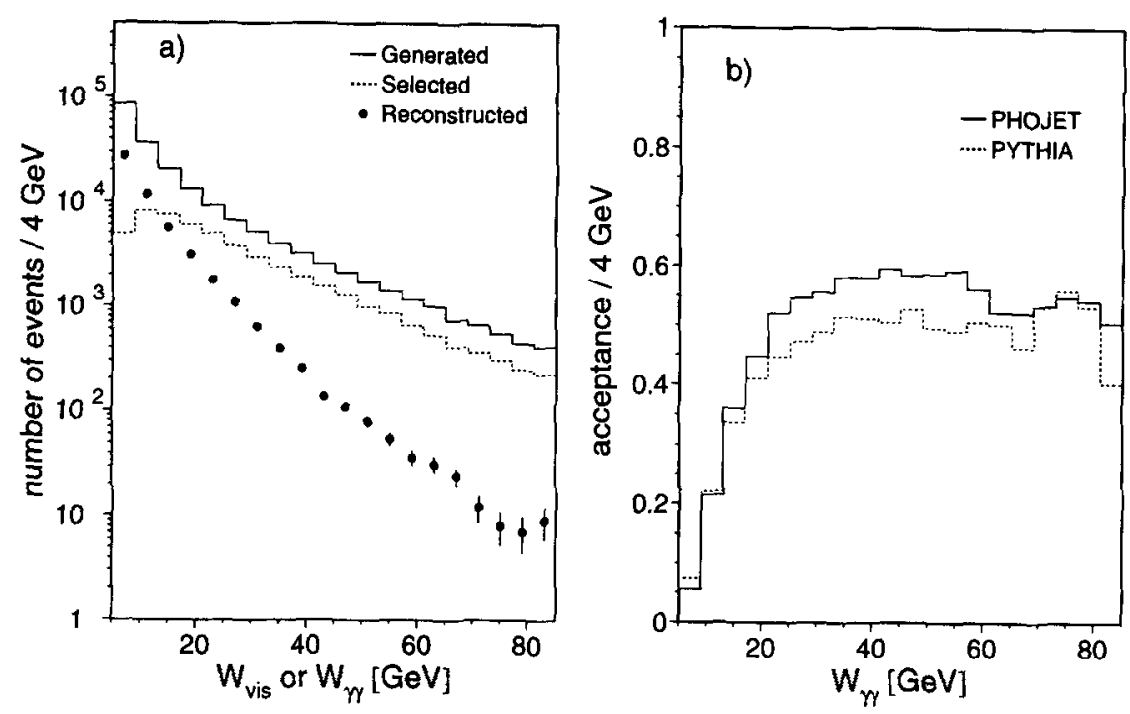

Fig. 7. (a)Distribution of the events generated with the PHOJET Monte Carlo at $\sqrt{s}=161 \mathrm{GeV}$ as a function of the $W_{y \gamma}$ mass before (continuous line) and after the selection cuts are applied (dashed line). The distribution of the selected events is distorted by the limited measurement of the mass $W_{\text {vis }}$ (dots with error bars). (b) Ratio of selected over generated events as a function of the two-photon mass, as calculated by the two generators PHOJET and PYTHIA
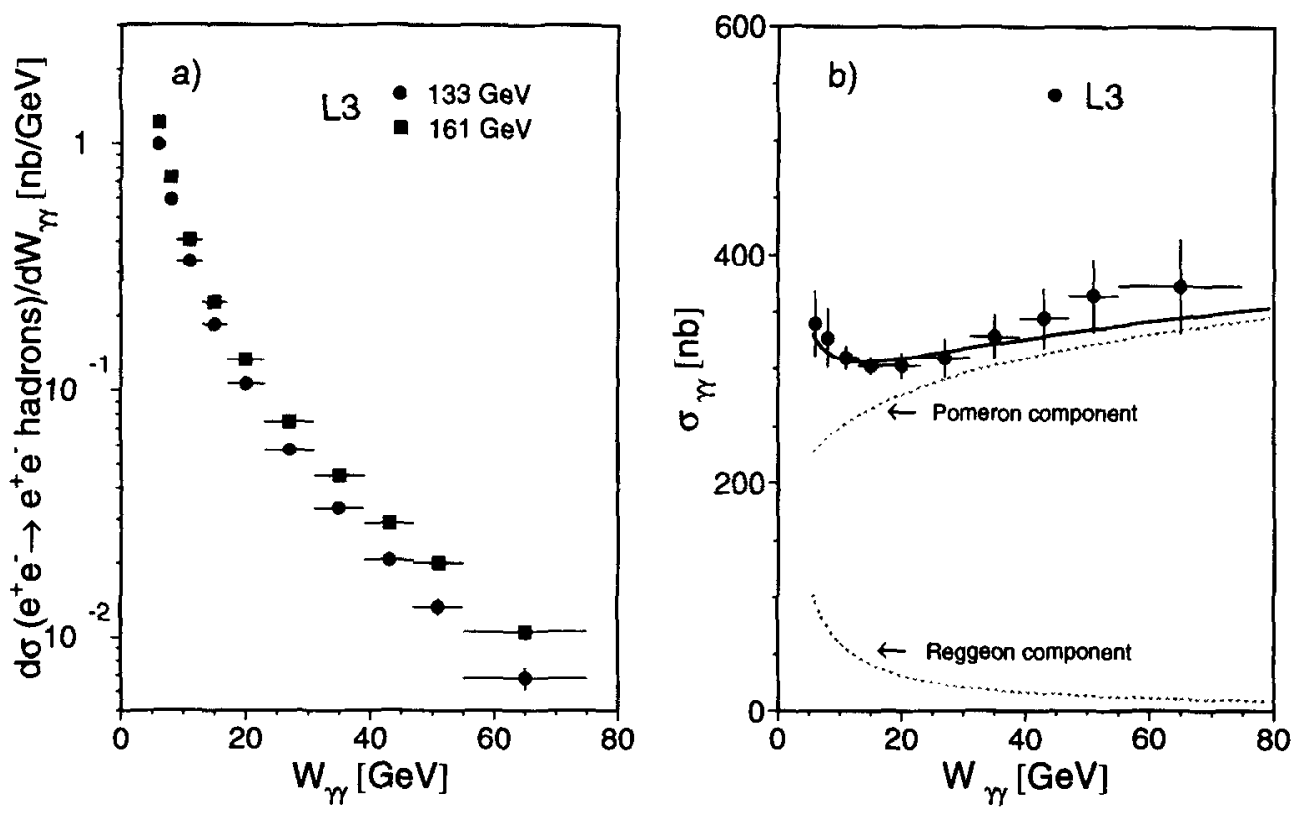

Fig. 8. a) The cross section $d \sigma\left(\mathrm{e}^{+} \mathrm{e}^{-} \rightarrow \mathrm{e}^{+} \mathrm{e}^{-}\right.$hadrons $) / d W_{\gamma \gamma}$ as measured at $\sqrt{s}=133 \mathrm{GeV}$ and at $\sqrt{s}=161 \mathrm{GeV}$. The errors are statistical and bin-to-bin systematic added in quadrature. An overall normalization systematic error of $\pm 6 \%$ is not included. b) Total cross section $\gamma \gamma \rightarrow$ hadrons. The errors are statistical and bin-to-bin systematic added in quadrature. An overall normalization systematic error of \pm $8 \%$ is not included. The continuous line is the Regge fit described in the text. The two components: the rapidly decreasing Reggeon part and the slow rising component due to Pomeron exchange are indicated with a dashed line. 
Table 2

The measured $d \sigma$ ( $\mathrm{e}^{+} \mathrm{e}^{-} \rightarrow \mathrm{e}^{+} \mathrm{e}^{-}$hadrons) cross sections as a function of the $\gamma \gamma$ centre of mass energy for the two sets of data. The $\sigma(\gamma \gamma \rightarrow$ hadrons $)$ is given for the combined data sample. The statistical errors, obtained after unfolding, and the bin-to-bin systematic errors are given. A global normalisation error of $6 \%$ must be added to all cross sections. A further normalisation error of $5 \%$, due to the uncertainty on the photon form factor, must be added to $\sigma(\gamma \gamma \rightarrow$ hadrons $)$.

\begin{tabular}{lllr}
\hline $\begin{array}{l}\Delta W_{\gamma \gamma} \\
(\mathrm{GeV})\end{array}$ & $\begin{array}{l}133 \mathrm{GeV} \\
d \sigma_{\mathrm{e}^{+} \mathrm{e}^{-}}(\mathrm{nb})\end{array}$ & $\begin{array}{l}161 \mathrm{GeV} \\
d \sigma_{\mathrm{e}^{+} \mathrm{e}^{-}}(\mathrm{nb})\end{array}$ & $\begin{array}{l}\text { All data } \\
\sigma_{\gamma \gamma}(\mathrm{nb})\end{array}$ \\
\hline $5-7$ & $1.980 \pm .050 \pm .102$ & $2.413 \pm .038 \pm .124$ & $340 \pm 4.6 \pm 29$ \\
$7-9$ & $1.173 \pm .030 \pm .045$ & $1.449 \pm .023 \pm .055$ & $327 \pm 4.4 \pm 26$ \\
$9-13$ & $1.329 \pm .027 \pm .021$ & $1.616 \pm .020 \pm .026$ & $310 \pm 3.3 \pm 10$ \\
$13-17$ & $0.733 \pm .018 \pm .013$ & $0.901 \pm .013 \pm .016$ & $303 \pm 3.8 \pm 8$ \\
$17-23$ & $0.634 \pm .016 \pm .018$ & $0.795 \pm .012 \pm .023$ & $303 \pm 3.8 \pm 11$ \\
$23-31$ & $0.458 \pm .013 \pm .023$ & $0.597 \pm .010 \pm .030$ & $310 \pm 4.4 \pm 17$ \\
$31-39$ & $0.266 \pm .010 \pm .014$ & $0.359 \pm .008 \pm .018$ & $329 \pm 6.0 \pm 19$ \\
$39-47$ & $0.164 \pm .008 \pm .011$ & $0.232 \pm .006 \pm .015$ & $345 \pm 7.9 \pm 26$ \\
$47-55$ & $0.106 \pm .006 \pm .008$ & $0.159 \pm .005 \pm .012$ & $364 \pm 10 . \pm 32$ \\
$55-75$ & $0.136 \pm .008 \pm .014$ & $0.211 \pm .006 \pm .021$ & $373 \pm 9.5 \pm 41$ \\
\hline
\end{tabular}

Table 3

The correlation matrix of the data after unfolding.

\begin{tabular}{|c|c|c|c|c|c|c|c|c|c|c|}
\hline$\Delta W_{\gamma \gamma}(\mathrm{GeV})$ & $5-7$ & $7-9$ & $9-13$ & $13-17$ & $17-23$ & $23-31$ & $31-39$ & $39-47$ & $47-55$ & $55-75$ \\
\hline $5-7$ & 1. & & & & & & & & & \\
\hline $7-9$ & .931 & 1. & & & & & & & & \\
\hline $9-13$ & .741 & .913 & 1. & & & & & & & \\
\hline $13-17$ & .506 & .710 & .908 & 1. & & & & & & \\
\hline $17-23$ & .331 & .506 & .730 & .910 & 1. & & & & & \\
\hline $23-31$ & .185 & .305 & .496 & .709 & .861 & 1. & & & & \\
\hline $31-39$ & .096 & .170 & .299 & .467 & .624 & .739 & 1. & & & \\
\hline $39-47$ & .052 & .093 & .172 & .292 & .424 & .545 & .558 & 1. & & \\
\hline $47-55$ & .030 & .055 & .107 & .185 & .278 & .379 & .418 & .378 & 1. & \\
\hline $55-75$ & .023 & .039 & .074 & .134 & .217 & .314 & .363 & .343 & .308 & 1. \\
\hline
\end{tabular}

very small in the central mass region. It has a maximum of $7 \%$ at $W_{\gamma \gamma}<10 \mathrm{GeV}$ and is $4 \%$ at $W_{\gamma \gamma}>50 \mathrm{GeV}$.

3. the differences due to the minimum number of tracks required in the analysis produce mainly normalisation shifts. The maximum bin-to-bin effect is $3 \%$ observed for $W_{\gamma \gamma}$ below $10 \mathrm{GeV}$. The overall normalisation uncertainty, arising from point 2 and 3 and evaluated separately for each beam energy, is estimated to be $\pm 6 \%$. Other uncertainties due to the analysis cuts are below the one per cent level and are neglected. The mass dependent contributions are added in quadrature in each $W_{\gamma \gamma}$ bin and are given as a systematic error in Table 2 .

To extract the total cross section of two real photons the photon flux $\mathcal{L}_{\gamma \gamma}$ [4] must be calculated and the hadronic two-photon processes must be extrapolated to zero $\mathrm{Q}^{2}$. This is done by considering the dominant transverse photon $(\mathrm{T})$ interaction as well as the small scalar photon (S) contribution [17]:

$$
\begin{aligned}
& d \sigma\left(\mathrm{e}^{+} \mathrm{e}^{-} \rightarrow \mathrm{e}^{+} \mathrm{e}^{-} \text {hadrons }\right) / d \tau \\
& \quad=\int \frac{d\left(Q_{1}^{2}\right)}{Q_{1}^{2}} \frac{d\left(Q_{2}^{2}\right)}{Q_{2}^{2}} \sum_{a, b=T, S} \mathcal{L}_{a b} \sigma_{a b}\left(W_{\gamma \gamma}, Q_{1}^{2}, Q_{2}^{2}\right)
\end{aligned}
$$

with the scaled variable $\tau=W_{y \gamma}^{2} / s$. The $W_{\gamma \gamma}$ and $Q^{2}$ dependencies of the cross section can be factorized for $Q^{2} \ell^{+} \ell^{-} W_{\gamma \gamma}^{2}$

$$
\begin{aligned}
& \sigma_{a, b}\left(W_{\gamma \gamma}, Q_{1}^{2}, Q_{2}^{2}\right) \\
& \quad=F_{a}\left(Q_{1}^{2}\right) F_{b}\left(Q_{2}^{2}\right) \sigma_{\gamma \gamma}\left(W_{\gamma \gamma}, 0 ., 0 .\right)
\end{aligned}
$$


For each $W_{\gamma \gamma}$ bin a numerical integration is performed over the bin width and over the unmeasured $Q^{2}$ of the scattered electron and positron. Many forms have been proposed for the $F\left(Q^{2}\right)$ form factors. The model [18], which adds a continuum contribution to a simple vector-meson dominance contribution, has been chosen for the central value calculation:

$$
\begin{aligned}
& F_{T}\left(Q^{2}\right)=\sum_{V} r_{V}\left(\frac{m_{V}^{2}}{m_{V}^{2}+Q^{2}}\right)^{2}+r_{c} \frac{m_{0}^{2}}{m_{0}^{2}+Q^{2}} \\
& F_{S}\left(Q^{2}\right)=\sum_{V} \xi \frac{Q^{2}}{m_{V}^{2}} r_{V}\left(\frac{m_{V}^{2}}{m_{V}^{2}+Q^{2}}\right)^{2}
\end{aligned}
$$

where $V=\rho, \omega, \phi, \xi=1 / 4, r_{\rho}=0.65, r_{\omega}=0.08$, $r_{\phi}=0.05$ and $r_{c}=1-r_{\rho}-r_{\omega}-r_{\phi}$.

Depending on the form factors used, this calculation may vary by $\pm 5 \%$ [17], independent of $W_{\gamma \gamma}$ in the mass range of this analysis.

The $\gamma \gamma \rightarrow$ hadrons cross sections thus obtained at $\sqrt{s}=133 \mathrm{GeV}$ and $\sqrt{s}=161 \mathrm{GeV}$ are compatible within statistical errors, the comparison giving a $\chi^{2}$ of 16 for the 10 measured points. The largest discrepancies are observed at low $W_{\gamma \gamma}$ values. The two measurements are therefore combined. Their weighted average is shown in Fig. $8 \mathrm{~b}$ and given in Table 2 together with the statistical and the bin-to-bin systematic errors. In the systematic errors the difference between the two samples has been added in quadrature to the systematic errors discussed above. In Fig. 9 our results for $5 \leq W_{y y} \leq 75 \mathrm{GeV}$ are shown together with the ones obtained in previous experiments [20] for $W_{\gamma \gamma} \leq 10$ $\mathrm{GeV}$. All measurements are displayed with their total systematic errors. For our data the normalisation systematic error of $\pm 6 \%$ plus the $\pm 5 \%$ uncertainty on the photon form factor are added in quadrature to the bin-to-bin error, displayed in the Figs. 8a and b.

\subsection{Regge parametrisation}

Total hadronic cross sections show a characteristic steep decrease in the region of low centre of mass energy followed by a slow rise at high energies. From Regge theory [7] this behaviour is understood as the consequence of the exchange of Regge trajectories, $\alpha(t)$, in the $t$-channel. The total cross section takes the form $\sigma_{\text {tot }} \propto s^{(\alpha(0)-1)}$. The low energy region is sensitive to Reggeon exchange ( $\mathrm{R}=\rho, \omega, \mathrm{f}, \mathrm{a} .$.$) ,$

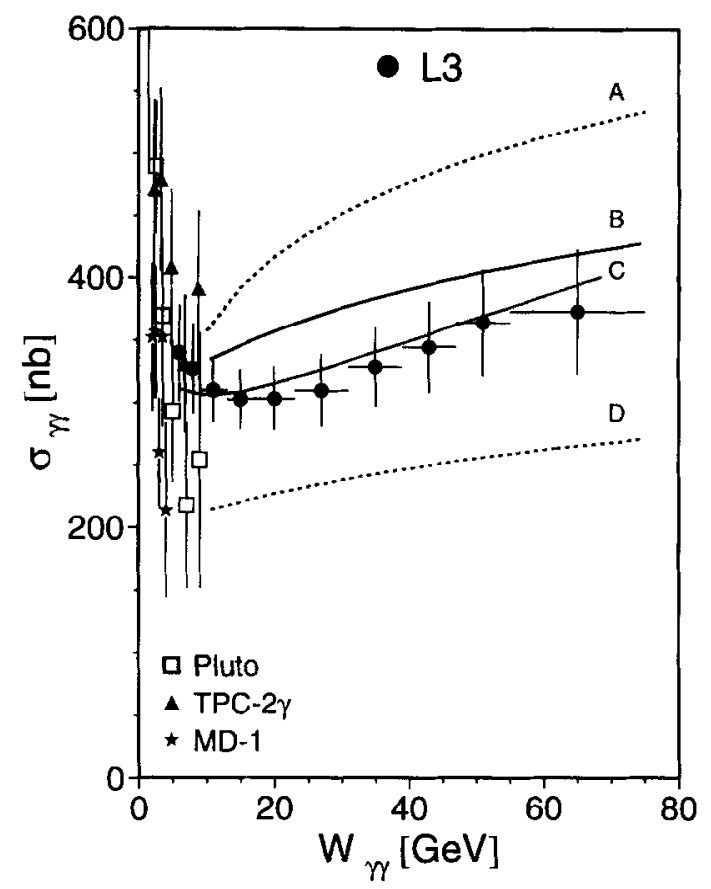

Fig. 9. The measured total cross section $\sigma(\gamma \gamma \rightarrow$ hadrons $)$ is compared to the best estimate by Schuler and Sjöstrand [1], line labelled as B, and to the predictions of the Dual Parton Model [2], labelled as $C$. The lower dashed line (D) represents the contribution of the VMD graph of Fig. Ib; the upper one (A) the maximum estimate of Ref. [1] compatible with photo-production data. Total errors, statistical and systematic added in quadrature, are drawn. For completeness the data of previous experiments [20] for $W_{\gamma \gamma}$ below $10 \mathrm{GeV}$ are included.

At high energies the Pomeron exchange dominates, $\alpha_{P}(0) \simeq 1$. Donnachie and Landshoff [8] showed that a parametrisation of the form

$\sigma_{\mathrm{tot}}=A s^{\epsilon}+B s^{-\eta}$

can account for the energy behaviour of all total cross sections, the powers of $s$ being universal. This is confirmed by the recent compilation of the total cross section data [19] where a fit of Eq. (5) for all hadron total cross sections gives a result compatible with a universal value of $\epsilon=0.0790 \pm 0.0011$ and $\eta=$ $0.4678 \pm 0.0059$. The coefficients $A$ and $B$ are process and $Q^{2}$ dependent. If photons behave predominantly like hadrons, this expression may also be valid for the two-photon total hadronic cross section. The data, with systematic bin-to-bin errors, are fitted to Eq. (6) with the parameters $\epsilon$ and $\eta$ fixed to the world average value. The coefficients $A$ and $B$ thus obtained are 
$A=173 \pm 7, \quad B=519 \pm 125, \quad \chi^{2} /$ d.o.f. $=3 / 8$

The correlation between $A$ and $B$ is -0.898 . The fit is shown in Fig. $8 \mathrm{~b}$ (continuous line) together with the Reggeon and the Pomeron components (dashed lines).

The cross sections predicted by Engel and Ranft [2] (line C in Fig. 9) are in good agreement with the data. In their model they use $\gamma p$ and $\mathrm{pp}$ data to fix the couplings of the Pomeron and of the Reggeon to the $q \bar{q}$ fluctuation of the photon. The cross sections are then calculated in the framework of a Dual Parton Model, with the unitarization constraint. Since there is a correlation between the VMD couplings and the Pomeron parameters, the predictions have an accuracy of $\pm 10 \%$ [2].

The model of Schuler and Sjöstrand [1] aims at a smooth superposition of hadron-like and point-like photon interactions. The fluctuation of both photons into vector mesons (Fig. 1b only) is not sufficient to describe the data (line D in Fig. 9). Adding the point-like splitting of the photon to $q \bar{q}$ pairs, the cross section increases (line B in Fig. 9). The maximum value, allowed by photo-production data, is indicated by the higher dashed line in Fig. 9.

\section{Conclusions}

In the two high energy runs of the LEP collider at $\sqrt{s}=133$ and $\sqrt{s}=161 \mathrm{GeV}$, a total of 32000 events of anti-tagged two-photon interaction $\mathrm{e}^{+} \mathrm{e}^{-} \rightarrow$ $\mathrm{e}^{+} \mathrm{e}^{-}$hadrons were observed in the L3 detector, with visible mass greater than $5 \mathrm{GeV}$.

The detailed features of the events: angular and momentum distributions, energy deposited in the calorimeters and visible mass are rather well reproduced by the model of the photon interactions contained in the recent generators PYTHIA and PHOJET.

The cross section $\sigma\left(\mathrm{e}^{+} \mathrm{e}^{-} \rightarrow \mathrm{e}^{+} \mathrm{e}^{-}\right.$hadrons $)$for $\left\langle Q^{2}\right\rangle \simeq 0.025 \mathrm{GeV}^{2}$ is measured in the interval $5 \leq$ $W_{\gamma \gamma} \leq 75 \mathrm{GeV}$. The real photon total cross section $\sigma(\gamma \gamma \rightarrow$ hadrons $)$ is also derived from the data. This is the first time the values of $W_{\gamma \gamma}$ above $10 \mathrm{GeV}$ are explored. The $\sigma(\gamma \gamma \rightarrow$ hadrons $)$ cross section is dominated by soft $\gamma \gamma$ interactions, where the photon behaves like a hadron. The increase with energy of this cross section is characteristic of Pomeron exchange.
The universal Regge parametrisation of A. Donnachie and P.V. Landshoff and the energy dependence fixed by the world average hadronic total cross sections reproduce well the data over the entire $W_{\gamma \gamma}$ range.

\section{Acknowledgements}

We wish to thank R. Engel for his continuous help in developing PHOJET, to adapt the program to the experimental conditions. We thank G.A. Schuler and T. Sjöstrand for their collaboration and useful discussions. We express our gratitude to the CERN accelerator divisions for the excellent performance of the LEP machine. We acknowledge with appreciation the effort of all engineers, technicians and support staff who have participated in the construction and maintenance of this experiment. Those of us who are not from member states thank CERN for its hospitality and help.

\section{References}

[1] G.A. Schuler and T. Sjöstrand, Nucl. Phys. B 407 (1993) 539;

G.A. Schuler and T. Sjöstrand, Z. Phys. C 73 (1997) 677.

[2] R. Engel, Z. Phys. C 66 (1995) 203;

R. Engel and J. Ranft, Phys. Rev. D 54 (1996) 4246;

R. Engel, private communication.

[3] T. Sjöstrand, Comput. Phys. Commun. 82 (1994) 74.

[4] V.M. Budnev et al., Physics Reports 15 (1974) 181.

[5] S. Jadach, B.F.L. Ward and Z.Was, Comput. Phys. Commun. 79 (1994) 503.

[6] F.A. Berends, P.H. Daverfeldt and R. Kleiss, Nucl. Phys. B 253 (1985) 441.

[7] P.D.B. Collins, Introduction to Regge theory (Cambridge U.P.,Cambridge, 1977).

[8] A. Donnachie and P.V. Landshoff, Phys. Lett. B 296 (1992) 227.

[9] L3 Coll., B. Adeva et al., Nucl. Instr. Meth. A 289 (1990) 35.

[10] M. Acciarri et al., Nucl. Instr. Meth. A 351 (1994) 300.

[11] P.Béné et al., Nucl. Instr. Meth. A 306 (1991) 150.

[12] R. Brun et al., GEANT 3.15 preprint CERN DD/EE/84-1 (Revised 1987).

[13] H. Fesefeldt, RWTH Aachen report PITHA 85/2 (1985).

[14] V. Blobel, Unfolding methods in high energy physics experiments 1984 CERN School of Computing CERN 85-09.

[15] G. D'Agostini, Nucl. Instr. Meth. A 362 (1995) 487.

[16] A. Höcker and V. Kertvelishvili, Nucl. Instr. Meth. A 372 (1996) 469 . 
[17] G.A. Schuler, Improving the equivalent-photon approximation in electron-positron collisions, hep-ph/9610406, CERNTH/96-297.

We wish to thank the author for providing us with the numerical integration program of the luminosity function.

[18] J.J. Sakurai and D. Schildknecht, Phys. Lett. B 40 (1972) 121.
[19] Review of Particle Physics, Phys. Rev. D 54 (1996) 192.

[20] PLUTO Coll., Ch. Berger et al., Phys. Lett. B 149 (1984) 421;

TPC $/ 2 \gamma$ Coll., H. Aihara et al., Phys. Rev. D 21 (1990) 2667;

MD1 Coll., S.E. Baru et al., Z. Phys C 53 (1992) 219. 